\title{
Machine Learning in Detection and Classification of Leukemia Using Smear Blood Images: A Systematic Review
}

\author{
Mustafa Ghaderzadeh (D), ${ }^{1}$ Farkhondeh Asadi $\left({ }^{D},{ }^{1}\right.$ Azamossadat Hosseini $(\mathbb{D}){ }^{1}$ \\ Davood Bashash (D), ${ }^{2}$ Hassan Abolghasemi $\left(D,{ }^{3}\right.$ and Arash Roshanpour ${ }^{4}$ \\ ${ }^{1}$ Department of Health Information Technology and Management, School of Allied Medical Sciences, \\ Shahid Beheshti University of Medical Sciences, Tehran, Iran \\ ${ }^{2}$ Department of Hematology and Blood Banking, School of Allied Medical Sciences, \\ Shahid Beheshti University of Medical Sciences, Tehran, Iran \\ ${ }^{3}$ Pediatric Congenital Hematologic Disorders Research Center, Shahid Beheshti University of Medical Sciences, Tehran, Iran \\ ${ }^{4}$ Department of Computer Science, Sama Technical and Vocational Training College, Tehran Branch (Tehran), \\ Islamic Azad University (IAU), Tehran, Iran
}

Correspondence should be addressed to Farkhondeh Asadi; asadifar@sbmu.ac.ir and Azamossadat Hosseini; souhosseini@ sbmu.ac.ir

Received 8 March 2021; Revised 13 May 2021; Accepted 27 May 2021; Published 25 June 2021

Academic Editor: Pengwei Wang

Copyright ( $) 2021$ Mustafa Ghaderzadeh et al. This is an open access article distributed under the Creative Commons Attribution License, which permits unrestricted use, distribution, and reproduction in any medium, provided the original work is properly cited.

\begin{abstract}
Introduction. The early detection and diagnosis of leukemia, i.e., the precise differentiation of malignant leukocytes with minimum costs in the early stages of the disease, is a major problem in the domain of disease diagnosis. Despite the high prevalence of leukemia, there is a shortage of flow cytometry equipment, and the methods available at laboratory diagnostic centers are timeconsuming. Motivated by the capabilities of machine learning (machine learning $(\mathrm{ML})$ ) in disease diagnosis, the present systematic review was conducted to review the studies aiming to discover and classify leukemia by using machine learning. Methods. A systematic search in four databases (PubMed, Scopus, Web of Science, and ScienceDirect) and Google Scholar was performed via a search strategy using Machine Learning (ML), leukemia, peripheral blood smear (PBS) image, detection, diagnosis, and classification as the keywords. Initially, 116 articles were retrieved. After applying the inclusion and exclusion criteria, 16 articles remained as the population of the study. Results. This review study presents a comprehensive and systematic view of the status of all published ML-based leukemia detection and classification models that process PBS images. The average accuracy of the ML methods applied in PBS image analysis to detect leukemia was $>97 \%$, indicating that the use of ML could lead to extraordinary outcomes in leukemia detection from PBS images. Among all ML techniques, deep learning (DL) achieved higher precision and sensitivity in detecting different cases of leukemia, compared to its precedents. ML has many applications in analyzing different types of leukemia images, but the use of ML algorithms to detect acute lymphoblastic leukemia (ALL) has attracted the greatest attention in the fields of hematology and artificial intelligence. Conclusion. Using the ML method to process leukemia smear images can improve accuracy, reduce diagnosis time, and provide faster, cheaper, and safer diagnostic services. In addition to the current diagnostic methods, clinical and laboratory experts can also adopt ML methods in laboratory applications and tools.
\end{abstract}

\section{Introduction}

Among all types of blood cancers, leukemia is the most common form of malignancy in different age groups, especially in children. This abnormal phenomenon is caused by excessive proliferation and immature growth of blood cells, which can damage red blood cells, bone marrow, and the defense system. In the United States, more than 3.5\% of new cancer cases are leukemia, and in 2018 alone, this country reported more than 60,000 new cases of this cancer. Malignant white blood cells, or lymphoblast, in the blood reach other organs, such as the spleen, brain, liver, and 
kidneys, and then metastasize to important tissues of the body $[1-3]$. There are different types of leukemia that hematologists in cell transplant laboratories can differentiate/ diagnose based on microscopic images. If the slide is correctly stained, some types of leukemia can be more easily identified and distinguished than others, but more equipment is needed to determine underlying leukemia. Figure 1 shows the stained slides of the most common different types of leukemia.

An early diagnosis of leukemia has always been a challenge to researchers, doctors, and hematologists. Enlargement of lymph nodes, pallor, fever, and weight loss are the symptoms of leukemia, but they can also be associated with other diseases. Leukemia diagnosis is difficult in its early stages due to the mild nature of the symptoms. The most common leukemia diagnosis method is the microscopic evaluation of PBS, but the golden standard for leukemia diagnosis only involves taking and analyzing bone marrow samples [3-6].

In the last two decades, various studies have adopted machine learning (ML) and computer-aided diagnostic methods for laboratory image analysis, hoping to overcome the limitations of a late leukemia diagnosis and determine its subgroups. These studies have analyzed blood smears images for diagnosing, differentiating, and counting the cells in various types of leukemia $[7,8]$.

ML is a well-known branch of artificial intelligence, comprising algorithms and mathematical relations, which was quickly introduced to the domain of clinical research. ML enables computers to be programmed without explicit experience and learns from that experience. The outcome of using these methods in medical data processing has been extraordinary, and they have made remarkable success in disease diagnosis [9-11]. Research indicates that, in medical image processing, ML methods greatly aid complex medical decision-making processes by extracting and then analyzing the features of these images [12-14]. As the number of medical diagnosis tools increased and a large volume of high-quality data was produced, there was an urgent need for more advanced data analysis methods. Traditional methods could not analyze such a large volume of data or find data patterns.

\section{Methodology}

The present systematic review aimed to identify the studies on leukemia detection and diagnosis by using ML techniques for peripheral blood smear (PBS) image analysis. The systematic search strategy was developed based on previous studies and the criteria selected by the authors.

2.1. Search Criteria. This study mainly aimed to answer the following questions:

(1) To what extent has ML been efficient in leukemia diagnosis and classification by using PBS images?
(2) Which ML algorithm has achieved high efficiency in PBS analysis?

(3) For the diagnosis and classification of what types of leukemia, has ML achieved better results?

(4) How can healthcare systems benefit from using ML methods for leukemia detection and diagnosis?

By surveying electronic databases that provide scientific articles on two domains of medicine and computer sciences, the researcher concluded that PubMed, Web of Science, Scopus, and ScienceDirect contain the highest number of articles relevant to the title and objectives of this study. The search was performed by using leukemia, leukemia diagnosis, and detection and ML keywords, based on the inclusion and exclusion criteria, from 2015 to 10 November 2020, and relevant articles were extracted from the said databases. EMBASE and IEEE databases were removed from the domain of search due to the similarity in publications. Table 1 lists the inclusion and exclusion criteria.

2.2. Data Extraction. By examining the previous articles, details of their methods and results were extracted and recorded in specially designed forms [15]. Two researchers extracted the data, and the disagreements were resolved upon discussions. The extracted data elements included the title of the article, country, year of publication, the studied population, ML technique, evaluation method, and results.

2.3. Quality Assessment. The quality of the eligible studies was assessed by the criteria proposed by Qiao [15]. The assessment was performed based on five categories: unmet need (limits in current non-ML approach), reproducibility (feature engineering methods, platforms/packages, hyperparameters), robustness (valid methods to overcome overfit, the stability of results), generalizability (external data validation), and clinical significance (predictors' explanation and suggested clinical use). A quality assessment table was provided by listing "yes" or "no" for the corresponding items in each category.

\section{Results}

A total of 116 articles were extracted from the four credible databases based on the search strategy. After reading the articles' abstract and full text, applying the inclusion and exclusion criteria, and selecting articles relevant to the title of the present study, 17 full-text articles were finally deemed eligible and were and selected. This process was performed based on the PRISMA flowchart (Figure 2). As ML methods and their applications in blood smear image analysis have newly emerged, this systematic search was conducted over the past five years. A review of the articles showed that, over time, the use of ML methods in PBS image analysis has expanded; seven articles in 2020, five articles in 2019, and 


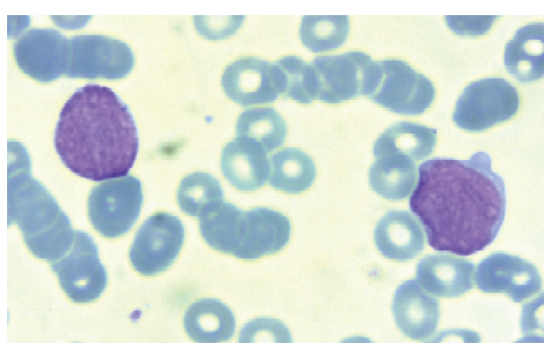

(a)

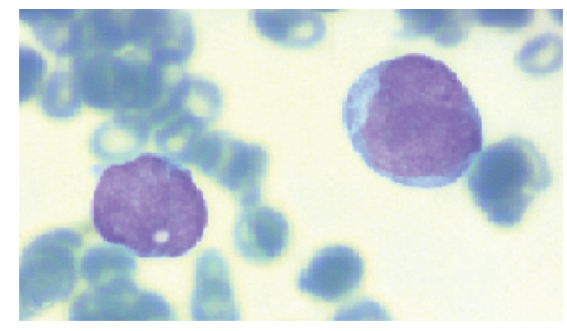

(c)

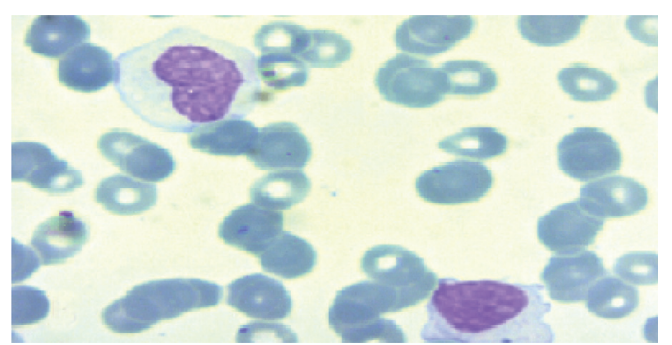

(b)

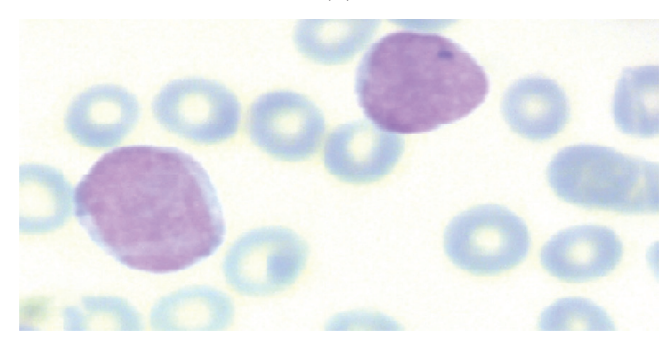

(d)

Figure 1: (a) AML (M1), (b) AML (M2), (c) B-ALL (pre-B), and (d) B-ALL (pro-B).

TABLE 1: Inclusion and exclusion criteria for review.

\begin{tabular}{ll}
\hline Inclusion criteria & Exclusion criteria \\
\hline (i) Machine learning & (i) DNA \\
(ii) Deep learning & (ii) RNA \\
(iii) Leukemia & (iii) Microarray \\
(iv) Blood smear image & (iv) Other diseases \\
(v) Microscopic WBC image & (v) Non-English reference abstracts were excluded \\
(vi) Full-text article available & (vi) Histology and molecular images \\
(vii) Written in English & (vii) Models not based on ML algorithms \\
(viii) Published after 2015 & (viii) Did not cover an original analysis (e.g., review, narrative) \\
(ix) Detection & (ix) Did not cover full details on methods \\
(x) Classification & \\
\hline
\end{tabular}

four articles in 2018 have focused on the diagnosis and classification of leukemia PBS images.

3.1. Leukemia Image Datasets. Diagnosis of leukemia in peripheral blood images is dependent on stained slide quality. Hence, a large number of quality standard datasets are not available. The majority of studies have employed published public datasets. To design and develop ML algorithms, hematologists have made some of these datasets (that include PBS images) available to researchers. ALL-IDB, one of the most well-known datasets published in two versions, has been utilized in many articles, most of which have diagnosed and classified acute lymphoblastic leukemia (ALL) via different ML techniques [16-21]. There is another published leukemia dataset called Benchmark for the development of ML algorithms, used by some studies. Most researchers have tested their proposed model only on homogeneous databases or private databases. However, a major challenge in a robust detection and classification model is the ability to diagnose the disease in databases with distinct characteristics [22]. Hence, to present a robust model and achieve reliable and valid results, some studies have employed a combination of these datasets as a crossdataset. Sharif has employed three datasets to achieve a system with high precision and efficiency in diagnosing various leukocytes [22]. Some researchers have also used local datasets in their studies. Among all types of leukemia diagnosed and classified by using ML, the most frequent type was ALL [23-26]. Figure 3 displays the diagnostic goals of various types of leukemia based on PBS image processing. In some articles, image analysis has been performed to count the leukocytes [19].

3.2. Overview of Machine Vision Techniques in PBS Image Analysis. Examining the methods adopted by the reviewed studies indicated that two categories of machine vision techniques have been used in PBS image analysis; machine learning and its important subclass, deep learning, are two categories of learning algorithms. The first strategy relies on selective image feature extraction. These methods are common in the extraction of a volume of image features via mathematical and ML algorithms. In this view, the goal of feature extraction is to obtain a set of image descriptors. By finding the relationship between these descriptors, the 




Figure 2: PRISMA flow diagram of the review process and exclusion of papers.

patterns determining the images can be discovered [17, 19]. Several classes of features have been considered by researchers and analyzed via ML algorithms to select the most valuable and most effective classification performance. The features extracted from the cytomorphological structure can include cell form, nucleus structure, chromatin, etc. Many articles consider other features as well. Table 2 summarizes the most common features in the field of blast analysis.

Al-jaboriy et al. used the nuclear-to-cytoplasmic ratio, nucleus compactness, nucleus form factors, nucleus eccentricity, nucleus elongation, and nucleus rigidity $[17,23,24,27]$. Among seven studies, which used traditional ML algorithm, four used the SVM method alone and with other algorithms $[18-20,24]$ and three utilized ANN and other algorithms [17]. Note that these algorithms are among the most popular algorithms in medical image processing.

The second view comprises methods in which feature extraction is performed automatically, and the researcher plays no role in feature selection. In these methods, building blocks of convolution neural network, including convolution and pooling layers, process the values corresponding to the pixels; in this way, features are extracted automatically
$[28,29]$. Then, the features are classified by feeding the features to a layer containing one or more classifiers. These methods extract important features and neglect less important ones. A review of the studies revealed that, to extract and process the features of PBS images for leukemia detection, many studies have employed the CNN algorithm and its state-of-the-art models [30-33]. The features of leukocytes by Vogado [22] simultaneously achieved using CaffeNet, AlexNet, and Vgg-f architectures, which, at that time, were among the most efficient CNN [22]. Figure 4 illustrates the frequency of use of both methods, ML and DL. The frequency of using ML for medical data analysis is daily increasing.

3.3. Segmentation in PBS Images. Segmentation is a common task in natural and medical image analysis. The researchers to achieve better classification rates use different types of segmentation. Segmentation is a method for image preprocessing applied for feature extraction and selection and could be considered as the first stage of feature extraction. Segmentation with the goal of extracting a cell from context 


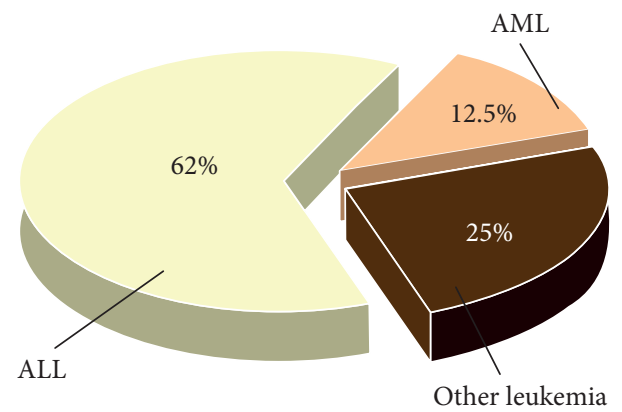

FIgure 3: The aim of studies in processing different types of leukemia PBS using ML.

TABLE 2: The most common features used in blast analysis using ML.

\begin{tabular}{|c|c|}
\hline Class of features & Parameters \\
\hline Color features & $\begin{array}{l}\text { (i) Mean color intensity for red component } \\
\text { (ii) Mean color intensity for green } \\
\text { component } \\
\text { (iii) Mean color intensity for blue } \\
\text { component } \\
\text { (iv) Mean color intensity for hue } \\
\text { component } \\
\text { (v) Mean color intensity for saturation } \\
\text { component } \\
\text { (vi) Mean color intensity for value } \\
\text { component }\end{array}$ \\
\hline Texture features & $\begin{array}{l}\text { (i) Homogeneity } \\
\text { (ii) Contrast } \\
\text { (iii) Correlation } \\
\text { (iv) Energy }\end{array}$ \\
\hline Statistical features & $\begin{array}{l}\text { (i) Mean } \\
\text { (ii) Variance } \\
\text { (iii) Skewness }\end{array}$ \\
\hline Wavelet features & $\begin{array}{l}\text { (i) Mean of HARR A coefficient } \\
\text { (ii) Mean of HARR H coefficient } \\
\text { (iii) Mean of HARR V coefficient } \\
\text { (iv) Variance of HARR A coefficient } \\
\text { (v) Variance of HARR H coefficient } \\
\text { (vi) Variance of HARR V coefficient }\end{array}$ \\
\hline $\begin{array}{l}\text { Morphological } \\
\text { features }\end{array}$ & $\begin{array}{l}\text { (i) Area, perimeter, roundness, elongation, } \\
\text { form factor, length-to-diameter ratio, } \\
\text { compactness, discrete Fourier transform }\end{array}$ \\
\hline
\end{tabular}



FIGURE 4: Different machine learning views in PBS image analysis.

or its nuclei from the cytoplasm provides an accurate view of the structure and features of the blast, and the ML technique by analysis the origin of the blast can identify leukemia or its subtypes. Several studies have diagnosed leukemia by segmenting and then extracting the features of some of these segments; other studies, however, have diagnosed leukemia without segmentation and merely by extracting the features from the entire image [12, 34-37].

3.3.1. Overview of Segmentation Techniques. Several studies trying to detect and differentiate leukocytes used ML techniques to segment and extract this cell and its nuclei from other blood cells. The main types of segmentation techniques include thresholding methods, boundary-based segmentation, region-based segmentation, and hybrid technology combining boundary and region standards, and most of the techniques combine boundary and region criteria [38-40]. Two techniques of blood smear image segmentation are more prominent and have received more attention from researchers. In the first view, which is based on the concept of thresholding and change color channels in the scope of cell sets, only the extraction of blasts without considering blasts feature is considered in research, and then the model is trained on these blasts [41]. In this method, the rest of the blood components like RBC are removed from the context of the images and, therefore, from the machine learning input. Al-jaboriy et al. using this type of segmentation removed all other blood components such as RBC cells and other erythrocyte lines and extracted only WBC cells, which include lymphocytes and lymphoblast. Figure 5 shows a view of this type of segmentation.

Another class of segmentation is object detection, in which segmentation is not performed from the edge of the cell, and the crop is done around the ROI surrounding the cell frame, accommodating other cellular components. In this type of segmentation, this entire box is fed to the model to learn its usage. This segmentation model has been used in many studies due to its high similarity between blood cells and their sensitivity to differentiation. This segmentation has been referred to as localization in some studies. In this type of segmentation, the noise components in the learning process are minimized. Figure 6 shows this type of segmentation. Other ML methods of segmentation are clustering [42], Gram-Schmidt orthogonalization method [43], edge detection, region growing [44], and optimization-based method [45]. In blood cell segmentation, more traditional ML algorithms have been used. 


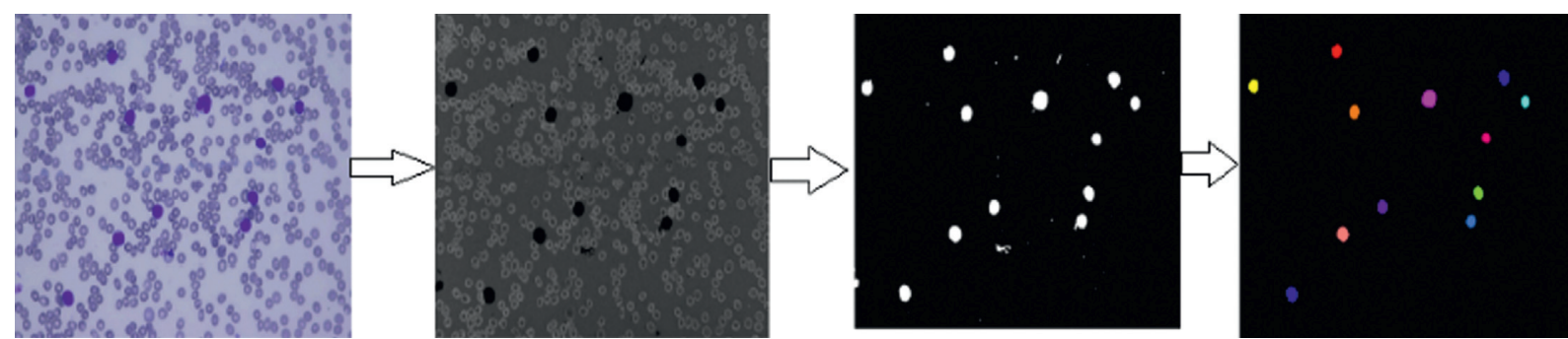

FIGURE 5: Localization, preprocessing, and thresholding segmentation [23].

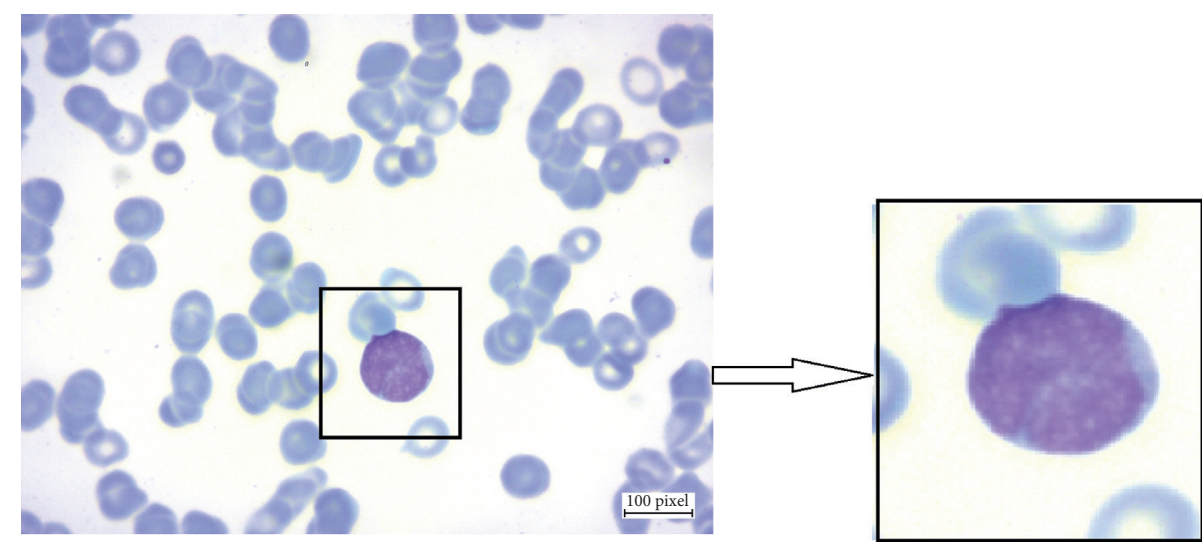

Figure 6: Blast segmentation-based object detection.

3.3.2. Overview of the ML Algorithm in Blood Cell Segmentation. Machine learning plays an important role in blood image segmentation, and segmentation is one of the first steps in identifying leukemia in blood smear images. Different machine learning algorithms have been used in most segmentation techniques. The purpose of cell segmentation is to identify the boundary between the nucleus and the cytoplasm for further characterization, such as the characterization of the nuclear properties, the properties of the cytoplasm, and the nuclear-to-cytoplasmic ratio, which is useful for explosive identification [39, 46, 47]. Many segmentation algorithms have been presented in the literature and the traditional ML algorithms based on selected features were the main and popular algorithms. Machine learning algorithms are used in the computational core of two categories of segmentation types. They are pixel-based image segmentation and region-based segmentation. Some other studies used shaped-based segmentation (threshold-based, edgebased, and region-based techniques) instead of regionbased segmentation. Among the different types of machine algorithms, clustering class algorithms had the most acceptance and efficiency. Kim et al. used clustering algorithms in the threshold, edge detection, pixel clustering, and region growing segmentation [48]. Kekre et al. used $\mathrm{k}$-mean and fuzzy c-mean algorithm vector quantization on the color pixel to segment the blood cells [44], and also Viswanathan used morphological contours (edge detection, erosion, and dilation) as features in the fuzzy c-mean algorithm to achieve a high-performance model in leukemia segmentation [46]. The other popular ML algorithm is watershed algorithm, which separating component-based morphological or other features presented in Table 2 treats pixels values as a local topography. The application of watershed segmentation to a distance map increases efficiency. Watershed segmentation is based on the idea of a catchment basin of a contour map. In other words, the water droplets follow the image gradient flow along the path to reach a local minimum. Many studies have used the watershed algorithm for segmentation. Using this algorithm has been easier and more acceptable than other algorithms [49-51]. Other ML algorithms such as SVM, ANN, and decision tree have been used frequently to segment blast in blood smear images. Table 3 lists the studies that have performed segmentation using the ML algorithm to extract blasts or their features for specific purposes, not just for leukemia detection or classification. Several of this research uses segmentation to extract nuclei of blast or other WBC cells.

Segmentation for leukemia detection or diagnosis is particularly much crucial. The accurate feature extraction and leukemia classification are proportionately dependent on the correct segmentation of the maximized and cropped lymphocytes. Table 4 presents the studies which have analyzed PBS images to diagnose (detect) or classify different kinds of leukemia based on the indicators considered in the present study. Some studies have merely diagnosed leukemia and aimed for its primary detection based on cell morphological changes $[19,21,22,25,26,75,76]$. 
TABLe 3: Studies that used ML algorithms for blast segmentation.

\begin{tabular}{|c|c|c|c|c|}
\hline Author & Type of feature extracted & ML segmentation technique & $\begin{array}{l}\text { No. of data } \\
\text { and details }\end{array}$ & $\begin{array}{l}\text { Performance } \\
\text { (accuracy \%) }\end{array}$ \\
\hline $\begin{array}{l}\text { Begum and Razak } \\
{[52]}\end{array}$ & $\begin{array}{l}\text { Morphological operations } \\
\text { erosion, dilation, opening, and } \\
\text { closing of nuclei }\end{array}$ & SVM & $\begin{array}{l}\text { Not } \\
\text { mentioned }\end{array}$ & $83 \%$ \\
\hline Jothi et al. [53] & $\begin{array}{l}\text { Morphological, wavelet, color, } \\
\text { texture, and statistical features, } \\
\text { and other features }\end{array}$ & $\begin{array}{l}\text { Naive Bayes, linear discriminant analysis, K- } \\
\text { nearest neighbor, support vector machine, } \\
\text { decision tree, and ensemble random under } \\
\text { sampling boost }\end{array}$ & 300 & $(60 \%-100 \%)$ \\
\hline $\begin{array}{l}\text { Gajul and Shelke } \\
{[54]}\end{array}$ & Not mentioned & K-mean clustering and morphological operations & 40 & - \\
\hline Vogado et al. [22] & Not identified & Automatic feature extraction & Three datasets & $99 \%$ \\
\hline Agaian et al. [55] & $\begin{array}{l}\text { Color, texture, shape, and } \\
\text { Hausdorff dimension feature }\end{array}$ & Using k-means clustering & 80 & $98 \%$ \\
\hline Negm et al. [51] & $\begin{array}{l}\text { Geometric, color, texture, and } \\
\text { size feature of blast }\end{array}$ & Using k-means clustering & 75 & $99.5 \%$ \\
\hline Su et al. [39] & Color and morphology features & $\begin{array}{l}\text { k-means cluster and constructing a cell image by } \\
\text { hidden Markov random field }\end{array}$ & 61 & $96 \%$ \\
\hline $\begin{array}{l}\text { Goutam and } \\
\text { Sailaja [56] }\end{array}$ & LDP feature & Using k-means clustering & 90 & $98 \%$ \\
\hline Shankar et al. [57] & Color, shape, and texture & Threshold by using the Zack algorithm & 33 & $96 \%$ \\
\hline Viswanathan [46] & $\begin{array}{c}\text { Morphological contour (edge } \\
\text { detection, erosion, and dilation) }\end{array}$ & Fuzzy c-means & - & $98 \%$ \\
\hline $\begin{array}{l}\text { Patel and Mishra } \\
{[58]}\end{array}$ & $\begin{array}{l}\text { Geometric, color, texture, and } \\
\text { size feature of cell }\end{array}$ & K-mean clustering and the Zack algorithm & 7 & $93 \%$ \\
\hline Zhao et al. [12] & $\begin{array}{l}\text { Morphological operation and } \\
\text { granularity feature are selected } \\
\text { automatically }\end{array}$ & $\mathrm{CNN}$ and SVM & 9 & $94 \%$ \\
\hline $\begin{array}{l}\text { Karthikeyan and } \\
\text { Poornima [59] }\end{array}$ & Geometrical, texture, and color & Fuzzy c-means & 19 & $90 \%$ \\
\hline $\begin{array}{l}\text { MoradiAmin et al. } \\
\text { [37] }\end{array}$ & $\begin{array}{l}\text { Geometrical and statistical } \\
\text { feature }\end{array}$ & Fuzzy c-means & 21 & $98 \%$ \\
\hline Rawat et al. [60] & Morphological operation & Global thresholding and morphological opining & 260 & $(79 \%-95.4 \%)$ \\
\hline Mishra et al. [61] & Texture and color & Marker-controlled watershed segmentation & 190 & $96 \%$ \\
\hline $\begin{array}{l}\text { Bhattacharjee and } \\
\text { Saini [36] }\end{array}$ & Morphological operation & $\begin{array}{l}\text { Morphological operations erosion, dilation, } \\
\text { opening, and closing }\end{array}$ & 120 & $96 \%$ \\
\hline $\begin{array}{l}\text { Khobragade et al. } \\
{[62]}\end{array}$ & Geometrical and statistical & Otsus's thresholding and Sobel operator & $\begin{array}{c}\text { Not } \\
\text { mentioned }\end{array}$ & $90 \%$ \\
\hline $\begin{array}{l}\text { Patil and Raskar } \\
\text { [41] }\end{array}$ & Color, shape, and texture & Thresholding by using Otsu's method & $\begin{array}{c}\text { Not } \\
\text { mentioned }\end{array}$ & $\begin{array}{c}\text { Not } \\
\text { mentioned }\end{array}$ \\
\hline Rawat et al. [34] & Shape features & Global thresholding and morphological opining & 420 & $96.75 \%$ \\
\hline Neelam et al. & Texture features & $\begin{array}{l}\text { K-mean clustering followed by expectation } \\
\text { maximization algorithm }\end{array}$ & $\begin{array}{c}\text { Not } \\
\text { mentioned }\end{array}$ & $80 \%$ \\
\hline Singh et al. [63] & Shape and texture features & ANN & $\begin{array}{l}\text { ALL-IDB (no: } \\
108)\end{array}$ & $97.2 \%$ \\
\hline $\begin{array}{l}\text { Singhal and Singh } \\
{[64]}\end{array}$ & Texture features & SVM & $\begin{array}{l}\text { ALL-IDB (no: } \\
\text { 260) }\end{array}$ & $93.8 \%$ \\
\hline Zhang et al. [65] & Shape features & Fuzzy system & $\begin{array}{l}\text { Local (not } \\
\text { mentioned) }\end{array}$ & $\begin{array}{c}\text { Not } \\
\text { mentioned }\end{array}$ \\
\hline Neoh et al. [66] & Shape, texture, and color features & Dempster-Shafer & $\begin{array}{l}\text { ALL-IDB (no: } \\
180)\end{array}$ & $96.7 \%$ \\
\hline Amin et al. [67] & Shape and texture features & SVM & Local (no: 21 ) & $97 \%$ \\
\hline Viswanathan [46] & Shape, color, and texture features & Fuzzy c-means classifier & $\begin{array}{l}\text { ALL-IDB (no: } \\
108)\end{array}$ & $98.0 \%$ \\
\hline
\end{tabular}


TABle 3: Continued.

\begin{tabular}{|c|c|c|c|c|}
\hline Author & Type of feature extracted & ML segmentation technique & $\begin{array}{l}\text { No. of data } \\
\text { and details }\end{array}$ & $\begin{array}{l}\text { Performance } \\
\text { (accuracy \%) }\end{array}$ \\
\hline $\begin{array}{l}\text { Bhattacharjee and } \\
\text { Saini [36] }\end{array}$ & Shape features & ANN & $\begin{array}{l}\text { ALL-IDB (no: } \\
120)\end{array}$ & $95.2 \%$ \\
\hline $\begin{array}{l}\text { ElDahshan et al. } \\
{[68]}\end{array}$ & Not mentioned & Field & $\begin{array}{l}\text { ALL-IDB (no: } \\
300)\end{array}$ & $\begin{array}{c}\text { Not } \\
\text { mentioned }\end{array}$ \\
\hline Rawat et al. [60] & Shape and texture features & SVM & $\begin{array}{l}\text { ALL-IDB (no: } \\
196)\end{array}$ & $89.8 \%$ \\
\hline Putzu et al. [35] & Shape, color, and texture features & SVM & $\begin{array}{l}\text { ALL-IDB (no: } \\
\text { 267) }\end{array}$ & $92.0 \%$ \\
\hline $\begin{array}{l}\text { Mohapatra et al. } \\
{[70]}\end{array}$ & Shape and texture features & Ensemble classifier & $\begin{array}{l}\text { Local dataset } \\
\text { (no: 104) }\end{array}$ & $94.7 \%$ \\
\hline Nasir et al. [71] & Shape and color features & MLP_BR & $\begin{array}{l}\text { Local dataset } \\
\text { (no: } 230)\end{array}$ & $95.7 \%$ \\
\hline $\begin{array}{l}\text { Mohapatra et al. } \\
{[72]}\end{array}$ & Shape and texture features & ANN & $\begin{array}{l}\text { Local dataset } \\
\text { (no: 100) }\end{array}$ & $\begin{array}{c}\text { Not } \\
\text { mentioned }\end{array}$ \\
\hline $\begin{array}{l}\text { Madhloom et al. } \\
{[73]}\end{array}$ & Shape and texture features & $\mathrm{kNN}$ clustering & $\begin{array}{l}\text { Local dataset } \\
\text { (no: 260) }\end{array}$ & $92.5 \%$ \\
\hline Pedreira et al. [74] & $\begin{array}{l}\text { Multiple clinical and laboratorial } \\
\text { features }\end{array}$ & ANN & $\begin{array}{l}\text { Local dataset } \\
\text { (no: 189) }\end{array}$ & $98.2 \%$ \\
\hline
\end{tabular}

TABle 4: Characteristics of studies that used machine learning algorithms in the detection and classification of blood smears.

First author,

year of

publication,

Aim of the study Data

ML method

Validation results

More information

and country

Al-jaboriy

et al., 2019,

ALL

Blood smear

segmentation images (ALL-IDB)

Accuracy $=97 \%$

The proposed model

Malaysia [17]

Al-Tahhan

et al., 2020,

-

Egypt [19]

Automatic

detection ALL

Blood smear
images (ALL-
IDB2)

KNN

SVM

ANN

SVM

ANN

detection of ALL images (local)

[24]

Boldú et al.,
2019, Spain
$[23]$

Dasariraju

et al., 2020,

USA [27]

Automatic
recognition of
different types of

blast

Detection and

classification of

immature

leukocytes for

diagnosis of

AML

\section{LDA}

RF

$$
\begin{gathered}
\text { Single-cell } \\
\text { morphological } \\
\text { dataset of } \\
\text { leukocytes from } \\
\text { AML patients and } \\
\text { nonmalignant } \\
\text { (public) }
\end{gathered}
$$

Fathi et al., 2018, Egypt

[16]
Classification of

ALL from

normal cases
Blood smear SVM with a Gaussian radial dataset (ALL-IDB) basis kernel
Accuracy $=96.2 \%$

Sensitivity $=97.3 \%$

Specificity $=95.3 \%$ detected 625 cells out of $540 \mathrm{WBC}$

Quadratic SVM has

the best performance

in detecting ALL

among ALL-IDB2

dataset

Artificial neural network has the best performance in detecting ALL

Classification accuracy for the six groups of cell types was 85.8

Segmentation, feature extraction, detection and classification, and calculation modules were applied

Goal of this research was to design a framework for classification of cancer based on medical images 
TABle 4: Continued.

First author,

year of
$\begin{aligned} & \text { publication, } \\ & \text { and country }\end{aligned}$

and country

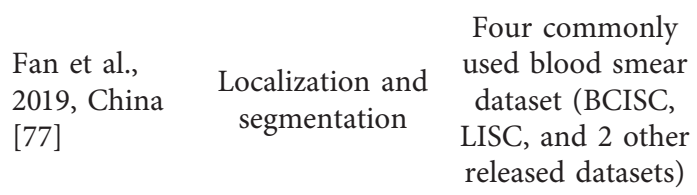

Dataset 1:

precision $=0.995 \%$

Dataset 2:

precision $=0.994 \%$

Dataset 3:

precision $=0.989 \%$

Dataset 4:

Proposed Leukocyte

Mask architecture to

gain best precision result with all datasets

precision $=0.984 \%$

Khilji et al.,

Proposed model

2020,

Bangladesh

Detection of ALL

ALL dataset

CNN-based different models

(C_NMC)

(encrypted)

Accuracy $=77.934 \%$ [75]
Nagiub

Abdelsalam

et al., 2018,

Egypt [26]
Detection of all

types of leukemia

Leukemia

microscopic

$\mathrm{CNN}$ compared with other state-of-the-art model and gain better accuracy

Different types of pretrained (CNN) models were applied and Inception-v3 model had the highest accuracy

Even deep neural networks: AlexNet,

Nagiub Abdelsalam et al., 2019, AML detection

AML microscopic images (local)

CNN (ResNet-101)

Accuracy $=100 \%$ Sensitivity $=100 \%$ GoogLeNet, VGG16,

VGG19, Inception-
Egypt [25]
Sparse-FCM and deep convolutional neural network
Accuracy $=93.5 \%$

Sensitivity $=95.28 \%$

Specificity $=93.89 \%$
Singh, 2020, and classification

India [78] of ALL

\section{ALL-IDB2} v3, ResNet50, and ResNet10

Grey Wolf-based Jaya optimization algorithm was applied for training $\mathrm{CNN}$

Sharif et al., 2020, Pakistan and Qatar different types of leukocytes
Recognition of
LISC, ALL-IDB1, and ALL-IDB2 malignant (public)
Localization using YOLOv2. Classification using PSO
Accuracy for ALL-

IDB1 $=97.2 \%$

Accuracy for ALLIDB2 $=100 \%$ Accuracy for LISC > 99\%
Naïve Bayes and discriminant analysis and particle swarm optimization was used

Goal of this research was to design a framework for classification of cancer based on medical images (3 architectures were used in feature extraction, SVM for classification)

Vogado et al., [22]
ALL-IDB1

ALL-IDB2

Leukocytes

CellaVision
CNN
$($ AlexNet + CaffeNet + Vgg-
f) and SVM

Accuracy $=100 \%$

Precision $=100 \%$
After detection, ALL subtype was classified based FAB classification system. Datasets

After segmentation, the nucleus of WBC cells different kinds of them was classified

\begin{tabular}{|c|c|c|c|c|c|}
\hline $\begin{array}{l}\text { Shafique and } \\
\text { Tehsin, 2020 } \\
\text { Pakistan [79] }\end{array}$ & $\begin{array}{l}\text { Detection and } \\
\text { classification of } \\
\text { ALL }\end{array}$ & $\begin{array}{l}\text { ALL-IDB1 and } \\
\text { ALL-IDB2 }\end{array}$ & CNN (AlexNet) & $\begin{array}{c}\text { accuracy }=99.50 \%, \text { ALL } \\
\text { subtype } \\
\text { classification }=96.06 \% \text {, } \\
\text { dataset precision }=0.984 \%\end{array}$ & $\begin{array}{l}\text { subtype was } \\
\text { classified based FAB } \\
\text { classification system. } \\
\text { Datasets }\end{array}$ \\
\hline $\begin{array}{l}\text { Hegde et al., } \\
2018, \text { India } \\
{[18]}\end{array}$ & $\begin{array}{l}\text { Detection of } \\
\text { nuclei and } \\
\text { classification of } \\
\text { WBC }\end{array}$ & Leishman & SVM & $\begin{array}{l}\text { Accuracy of detection of } \\
\text { lymphocyte }=100 \%\end{array}$ & $\begin{array}{l}\text { After segmentation, } \\
\text { the nucleus of WBC } \\
\text { cells different kinds } \\
\text { of them was classified }\end{array}$ \\
\hline
\end{tabular}


TABLE 4: Continued.

\begin{tabular}{|c|c|c|c|c|c|}
\hline $\begin{array}{l}\text { First author, } \\
\text { year of } \\
\text { publication, } \\
\text { and country }\end{array}$ & Aim of the study & Data & ML method & Validation results & More information \\
\hline $\begin{array}{l}\text { Jha and Dutta, } \\
\text { 2019, India } \\
{[21]}\end{array}$ & Detection of ALL & ALL-IDB2 & $\begin{array}{c}\text { Proposed hybrid } \\
\text { segmentation + Chrono- } \\
\text { SCA-ACNN }\end{array}$ & $\begin{aligned} \text { Accuracy } & =99 \% \\
\text { Sensitivity } & =100 \%\end{aligned}$ & $\begin{array}{c}\text { Nucleus and } \\
\text { cytoplasm } \\
\text { segmentation using } \\
\text { Chrono-SCA-ACNN } \\
\text { (chronological sine } \\
\text { cosine algorithm- } \\
\text { based actor-critic } \\
\text { neural network) }\end{array}$ \\
\hline
\end{tabular}

\section{Discussion}

Microscopic evaluation of PBS images is the most common primary method of leukemia diagnosis in its early stages. Still, a manual examination of these smears can cause errors in determining the type of the disease and lead to nonstandard reports. Moreover, the examination of these smears is tiresome and time-consuming, thus influencing the diagnostic precision. Accordingly, there is a need for an automatic method to provide a precise diagnosis, without being affected by the technicians' experience or the operator's fatigue and job pressures [49, 80].

Upon a search in scientific databases, it was found that no comprehensive systematic review had been conducted on PBS image analysis via ML methods. Therefore, the authors conducted a review study on the applications of ML in the diagnosis and classification of different types of leukemia based on PBS images. By comparing the previous studies, the present research answered the questions posed by the researcher at the outset.

In terms of smear preparation, several factors (e.g., illumination condition, staining time, blood film thickness, and a defect in the film) lead to undesirable visual artifacts or different color distributions in the laboratory images. These issues complicate the precise detection and monitoring of blood smears. As processing these smear images by ML is problematic, preprocessing is necessary [81]. As for leukemia detection using ML algorithms, data preprocessing (e.g., preparation, normalization, and segmentation) can promote the precision of leukemia detection. For precise leukemia detection with minimum error via ML methods, it is suggested that a set of preprocessing techniques be adopted for dataset preparation.

The selection of effective features is the bedrock of preliminary processing of blood smears via ML methods. In cases where the researcher could control the selection and analysis of blood cell features, the main problem was selecting these features to determine leukemia. Some studies have used color and shape, while others have utilized texture and different texture metrics as the features of blast cells. The manual selection of the most important features is always associated with some degree of error, and this process is always viewed as a major challenge. Medical texts have not mentioned any of these features selected by manual methods as a definitive method for leukemia differential diagnosis $[1,24,82]$. Thus, the selection of several important features from among a large number of features is a completely algorithmic process, and promoting the efficiency of feature selection depends on the algorithm's method. The studies demonstrated that methods extracting fewer cell features have attained a lower precision in leukemia diagnosis. It seems that, to achieve better results in leukemia detection and diagnosis, one can adopt feature extraction methods based on hybrid algorithms or swarm intelligence and pay attention to further coverage of the feature space. It is also recommended that a set of various features, including geometrical, statistical, and morphological ones, be used for leukemia detection. ML methods require manual feature extraction and selection; if the number of images is acceptable for DL, instead of ML, it is better to use the DL method owing to its mechanism.

A major problem associated with leukemia diagnosis via ML algorithms in different studies is the lack of comprehensive datasets of leukemia smear images, an issue which causes problems for the ML methods, e.g., overfitting. Based on the studies, and with respect to the data-driven nature of these methods, one can show that diagnostic errors are higher in the case of smaller datasets. This is why the results of many studies cannot be confirmed because small/local datasets have been used. Thus, to have a robust ML method for leukemia diagnosis/classification, a comprehensive dataset with sufficient data is required, yet the datasets existing in the reviewed studies did not satisfy this basic need. Of course, there are techniques for increasing the data, which, by processing the main images, create new images that maintain the features of the main images. To overcome this problem in DL, numerous studies have reported that augmentation techniques can lead to better results in terms of pattern recognition [47-49]. It seems that image augmentation can lead to better coverage of data space and markedly improve the results of leukemia detection by using these methods. Based on the review of previous studies and the results of smear processing, it can be concluded that ML methods and techniques have received more attention for the diagnosis and classification of acute leukemia, whether AML or ALL, compared to other types. No comprehensive study has examined the performance of traditional and visual leukemia diagnosis by using smear images. However, 
studies that have diagnosed leukemia via ML techniques have achieved extraordinary results, with a disease detection mean accuracy of $>96 \%$. Although the applications of machine learning in disease diagnosis and blood cell imaging are still evolving, the use of these algorithms in cell counting and blood cell type differentiation is expanding in the healthcare industry. Nowadays, the use of cell counter devices to determine and count blood components based on $\mathrm{ML}$ is becoming more common. It is thought that, in the near future, bone marrow transplant laboratories could replace traditional devices with applications and software based on ML, especially DL, to offer a timely method and assist a diagnosis with high certainty and low detection error in the early stages.

\section{Conclusion}

Blood smear image analysis is a vital role in the diagnosis of many blood-related diseases. The diagnosis of leukemia in its early stages and the first smears can lead to immediate diagnosis and the quick initiation of the treatment. Blood smear image analysis by ML methods can aid the diagnosis of early-onset leukemia and the determination of subtypes with a minimum error at the shortest time, so that the process of treatment can be immediately started. A promising future direction for research can be the application of novel ML algorithms, in particular, DL, in computer-aided detection (CAD) systems, whole-slide imaging (WSI), and even apps and software at hematology laboratories, to help the pathologists and oncologists in better detecting leukemia. In the 2018 meeting of the American Society of Hematology, Höllein et al. investigated 43 roles of AI in MFC for B cell lymphoma and leukemia diagnosis. By using the data of 38416 patients and control groups, a model was developed by using neural networks. This system achieved 97\% precision in determining normal and abnormal cells. Still, the precision of B cell lymphoma and leukemia classification was $74 \%$. Thus, it is recommended that, in the near future, the use of ML algorithms for the analysis of blood smear images progresses from the phase of modeling to the phase of implementation.

\section{Data Availability}

No data were used to support the findings of this study.

\section{Ethical Approval}

This study was approved by Iran National Committee for Ethics in Biomedical Research with Approval ID IR.SBMU.RETECH.REC.1399.735.

\section{Disclosure}

This study was part of a $\mathrm{PhD}$ project conducted at Shahid Beheshti University of Medical Sciences, Tehran, Iran.

\section{Conflicts of Interest}

The authors declare that they have no conflicts of interest.

\section{References}

[1] R. B. Hegde, K. Prasad, H. Hebbar, B. M. K. Singh, and I. Sandhya, "Automated decision support system for detection of leukemia from peripheral blood smear images," Journal of Digital Imaging, vol. 33, pp. 361-374, 2019.

[2] "Hematology TAS of facts-and-statistics," 2018, http://www. hematology.org/.

[3] S. M. Namayandeh, Z. Khazaei, M. Lari Najafi, E. Goodarzi, and A. Moslem, "GLOBAL Leukemia in children 0-14 statistics 2018, incidence and mortality and human development index (HDI): GLOBOCAN sources and methods," Asian Pacific Journal of Cancer Prevention, vol. 21, no. 5, pp. 1487-1494, 2020.

[4] O. Wolach and R. M. Stone, "Mixed-phenotype acute leukemia," Current Opinion in Hematology, vol. 24, no. 2, pp. 139-145, 2017.

[5] C.-H. Pui, "Childhood leukemias," New England Journal of Medicine, vol. 332, no. 24, pp. 1618-1630, 1995.

[6] "Childhood leukemias," 2020, https://www.nejm.org/doi/full/ 10.1056/NEJM199506153322407.

[7] F. Xing and L. Yang, "Robust nucleus/cell detection and segmentation in digital pathology and microscopy images: a comprehensive review," IEEE Reviews in Biomedical Engineering, vol. 9, pp. 234-263, 2016.

[8] J. Wen, Y. Xu, Z. Li, Z. Ma, and Y. Xu, "Inter-class sparsity based discriminative least square regression," Neural Networks, vol. 102, pp. 36-47, 2018.

[9] Z. Obermeyer and E. J. Emanuel, "Predicting the future-big data, machine learning, and clinical medicine," New England Journal of Medicine, vol. 375, no. 13, pp. 1216-1219, 2016.

[10] V. Ehrenstein, H. Nielsen, A. B. Pedersen, S. P. Johnsen, and L. Pedersen, "Clinical epidemiology in the era of big data: new opportunities, familiar challenges," Clinical Epidemiology, vol. 9, pp. 245-250, 2017.

[11] M. Ghaderzadeh, F. Rebecca, and A. Standring, "Comparing performance of different neural networks for early detection of cancer from benign hyperplasia of prostate," Applied Medical Informatics, vol. 33, pp. 45-54, 2013.

[12] J. Zhao, M. Zhang, Z. Zhou, J. Chu, and F. Cao, "Automatic detection and classification of leukocytes using convolutional neural networks," Medical \& Biological Engineering \& Computing, vol. 55, no. 8, pp. 1287-1301, 2017.

[13] I. Oikonomidis, N. Kyriazis, and A. A. Argyros, "Efficient model-based 3D tracking of hand articulations using Kinect," in Proceedings of the 22nd British Machine Vision Conference, BMVC'2011, Dundee, UK, 2011.

[14] T. W. Reader, R. Flin, K. Mearns, and B. H. Cuthbertson, "Developing a team performance framework for the intensive care unit," Critical Care Medicine, vol. 37, no. 5, pp. 17871793, 2009.

[15] N. Qiao, "A systematic review on machine learning in sellar region diseases: quality and reporting items," Endocrine Connections, vol. 8, no. 7, pp. 952-960, 2019.

[16] E. Fathi, M. J. Rezaee, R. Tavakkoli-Moghaddam, A. Alizadeh, and A. Montazer, "Design of an integrated model for diagnosis and classification of pediatric acute leukemia using 
machine learning," Proceedings of the Institution of $\mathrm{Me}$ chanical Engineers, Part H: Journal of Engineering in Medicine, vol. 234, no. 10, pp. 1051-1069, 2020.

[17] S. S. Al-jaboriy, N. N. A. Sjarif, S. Chuprat, W. M. Abduallah, and W. M. Abduallah, "Acute lymphoblastic leukemia segmentation using local pixel information," Pattern Recognition Letters, vol. 125, pp. 85-90, 2019.

[18] R. B. Hegde, K. Prasad, H. Hebbar, and B. M. K. Singh, "Development of a robust algorithm for detection of nuclei and classification of white blood cells in peripheral blood smear images," Journal of Medical Systems, vol. 42, no. 6, p. 110, 2018.

[19] F. E. Al-Tahhan, M. E. Fares, A. A. Sakr, and D. A. Aladle, "Accurate automatic detection of acute lymphatic leukemia using a refined simple classification," Microscopy Research \& Technique, vol. 83, no. 10, pp. 1178-1189, 2020.

[20] E. M. F. E. Houby, "Framework of computer aided diagnosis systems for cancer classification based on medical images," Journal of Medical Systems, vol. 42, p. 157, 2018.

[21] K. K. Jha and H. S. Dutta, "Nucleus and cytoplasm-based segmentation and actor-critic neural network for acute lymphocytic leukaemia detection in single cell blood smear images," Medical \& Biological Engineering \& Computing, vol. 58, pp. 171-186, 2019.

[22] L. H. S. Vogado, R. M. S. Veras, F. H. D. Araujo, R. R. V. Silva, and K. R. T. Aires, "Leukemia diagnosis in blood slides using transfer learning in CNNs and SVM for classification," Engineering Applications of Artificial Intelligence, vol. 72, pp. 415-422, 2018.

[23] L. Boldú, A. Merino, S. Alférez, A. Molina, A. Acevedo, and J. Rodellar, "Automatic recognition of different types of acute leukaemia in peripheral blood by image analysis," vol. 72, no. 11, pp. 755-761, 2019.

[24] A. Bodzas, P. Kodytek, and J. Zidek, "Automated detection of acute lymphoblastic leukemia from microscopic images based on human visual perception," Frontiers in Bioengineering and Biotechnology, vol. 8, pp. 1005-1013, 2020.

[25] E. M. Nagiub Abdelsalam, K. F. Hussain, N. M. Omar, and Q. T. Ali, "Acute myeloid leukemia diagnosis using deep learning," Clinical Lymphoma Myeloma and Leukemia, vol. 19, p. S206, 2019.

[26] E. M. Nagiub Abdelsalam, K. F. Hussain, N. M. Omar, Q. T. Ali, T. Dao, and D. Scheinberg, "RES-069 improved sensitivity and specificity for mutated and unmutated class I HLA ligand identifications lead to better immunotherapy target discovery RES-064 computer aided leukemia detection using microscopic blood image based machine learning," Clinical Lymphoma Myeloma and Leukemia, vol. 18, p. S297, 2018.

[27] S. Dasariraju, M. Huo, and S. Mccalla, "Detection and classification of immature leukocytes for diagnosis of acute myeloid leukemia using random forest algorithm," Bioengineering (Basel), vol. 7, no. 4, p. 120, 2020.

[28] M. Ghaderzadeh, F. Asadi, R. Jafari, D. Bashash, H. Abolghasemi, and M. Aria, "Deep convolutional neural network-based computer-aided detection system for COVID19 using multiple lung scans: design and implementation study," Journal of Medical Internet Research, vol. 23, no. 4, Article ID e27468, 2021.

[29] M. Ghaderzadeh and F. Asadi, "Deep learning in the detection and diagnosis of COVID-19 using radiology modalities: a systematic review," Journal of Healthcare Engineering, vol. 2021, Article ID 6677314, 10 pages, 2021.
[30] S. Koitka and C. M. Friedrich, "Traditional feature engineering and deep learning approaches at medical classification task of ImageCLEF 2016," 2016, http://ceur-ws.org/Vol-1609/ 16090304.pdf.

[31] L. Oakden-Rayner, G. Carneiro, T. Bessen, J. C. Nascimento, A. P. Bradley, and L. J. Palmer, "Precision radiology: predicting longevity using feature engineering and deep learning methods in a radiomics framework," Scientific Reports, vol. 7, pp. 1-13, 2017.

[32] G. E. Hinton and R. R. Salakhutdinov, "Reducing the dimensionality of data with neural networks," Science, vol. 313, pp. 504-507, 2006.

[33] Y. LeCun, Y. Bengio, and G. Hinton, “Deep learning," Nature, vol. 521, no. 7553, pp. 436-444, 2015.

[34] J. Rawat, A. Singh, H. S. Bhadauria, J. Virmani, and J. S. Devgun, "Computer assisted classification framework for prediction of acute lymphoblastic and acute myeloblastic leukemia," Biocybernetics and Biomedical Engineering, vol. 37, no. 4, pp. 637-654, 2017.

[35] L. Putzu, G. Caocci, and C. Di Ruberto, "Leucocyte classification for leukaemia detection using image processing techniques," Artificial Intelligence in Medicine, vol. 62, no. 3, pp. 179-191, 2014.

[36] R. Bhattacharjee and L. M. Saini, "Robust technique for the detection of acute lymphoblastic leukemia," in Proceedings of the 2015 IEEE Power, Communication and Information Technology Conference (PCITC), Bhubaneswar, India, 2015.

[37] M. MoradiAmin, N. Samadzadehaghdam, S. Kermani, and A. Talebi, "Enhanced recognition of acute lymphoblastic leukemia cells in microscopic images based on feature reduction using principle component analysis," Frontiers in Biomedical Technologies, vol. 2, pp. 128-136, 2015.

[38] K. Krishnaveni, S. Allwin, S. P. K. Kenny, and G. Mariappan, "Analysis for textural features in nuclei of cervical cyto images," in Proceedings of the 2010 IEEE International Conference on Computational Intelligence and Computing Research, Coimbatore, India, 2010.

[39] J. Su, S. Liu, and J. Song, "A segmentation method based on HMRF for the aided diagnosis of acute myeloid leukemia," Computer Methods and Programs in Biomedicine, vol. 152, pp. 115-123, 2017.

[40] F. Scotti, "Robust segmentation and measurements techniques of white cells in blood microscope images," in Proceedings of the 2006 IEEE Instrumentation and Measurement Technology Conference, Sorrento, Italy, 2006.

[41] T. G. Patil and V. B. Raskar, "Automated leukemia detection by using contour signature method," International Journal of Advance Foundation and Research in Computer, vol. 2, 2015.

[42] H. Sheikh, B. Zhu, and E. Micheli-Tzanakou, "Blood cell identification using neural networks," in Proceedings of the 22nd Annual Northeast Bioengineering Conference, New Brunswick, NJ, USA, 1996.

[43] S. H. Rezatofighi and H. Soltanian-Zadeh, "Automatic recognition of five types of white blood cells in peripheral blood," Computerized Medical Imaging and Graphics, vol. 35, no. 4, pp. 333-343, 2011.

[44] H. Kekre, B. Archana, and H. R. Galiyal, "Segmentation of blast using vector quantization technique," International Journal of Computer Applications, vol. 72, no. 15, 2013.

[45] E. Cuevas, M. Díaz, M. Manzanares, D. Zaldivar, and M. Perez-Cisneros, "An improved computer vision method for white blood cells detection," Computational and Mathematical Methods in Medicine, vol. 2013, Article ID 137392, 14 pages, 2013. 
[46] P. Viswanathan, "Fuzzy C means detection of leukemia based on morphological contour segmentation," Procedia Computer Science, vol. 58, pp. 84-90, 2015.

[47] Y. Li, R. Zhu, L. Mi, Y. Cao, and D. Yao, "Segmentation of white blood cell from acute lymphoblastic leukemia images using dual-threshold method," Computational and Mathematical Methods in Medicine, vol. 2016, Article ID 9514707, 12 pages, 2016.

[48] K. Kim, J. Jeon, W. Choi, P. Kim, and Y.-S. Ho, "Automatic cell classification in human's peripheral blood images based on morphological image processing," in Proceedings of the 14th Australian Joint Conference on Artificial Intelligence, Adelaide, Australia, 2001.

[49] E. A. Mohammed, M. M. A. Mohamed, C. Naugler, and B. H. Far, "Chronic lymphocytic leukemia cell segmentation from microscopic blood images using watershed algorithm and optimal thresholding," in Proceedings of the 2013 26th IEEE Canadian Conference on Electrical and Computer Engineering (CCECE), Regina, Canada, 2013.

[50] J. Hari, A. S. Prasad, and S. K. Rao, "Separation and counting of blood cells using geometrical features and distance transformed watershed," in Proceedings of the 2014 2nd International Conference on Devices, Circuits and Systems (ICDCS), Coimbatore, India, 2014.

[51] A. S. Negm, O. A. Hassan, and A. H. Kandil, "A decision support system for acute leukaemia classification based on digital microscopic images," Alexandria Engineering Journal, vol. 57, no. 4, pp. 2319-2332, 2018.

[52] A. R. Begum and T. A. Razak, "A proposed novel method for detection and classification of leukemia using blood microscopic images," International Journal of Advanced Research in Computer Science, vol. 8, 2017.

[53] G. Jothi, H. H. Inbarani, A. T. Azar, and K. R. Devi, “Rough set theory with Jaya optimization for acute lymphoblastic leukemia classification," Neural Computing and Applications, vol. 31, no. 9, pp. 5175-5194, 2019.

[54] Y. A. Gajul and R. Shelke, "Computerized detection system for acute myelogenous leukemia in blood microscopic images," International Journal of Innovative Research in Science, Engineering and Technology, vol. 5, no. 6, pp. 10073-10079, 2016.

[55] S. Agaian, M. Madhukar, and A. T. Chronopoulos, "Automated screening system for acute myelogenous leukemia detection in blood microscopic images," IEEE Systems Journal, vol. 8, no. 3, pp. 995-1004, 2014.

[56] D. Goutam and S. Sailaja, "Classification of acute myelogenous leukemia in blood microscopic images using supervised classifier," in Proceedings of the 2015 IEEE International Conference on Engineering and Technology (ICETECH), Cimbatore, India, 2015.

[57] V. Shankar, M. M. Deshpande, N. Chaitra, and S. Aditi, "Automatic detection of acute lymphoblasitc leukemia using image processing," in Proceedings of the 2016 IEEE International Conference on Advances in Computer Applications (ICACA), Coimbatore, India, 2016.

[58] N. Patel and A. Mishra, "Automated leukaemia detection using microscopic images," Procedia Computer Science, vol. 58, pp. 635-642, 2015.

[59] T. Karthikeyan and N. Poornima, "Microscopic image segmentation using fuzzy c means for leukemia diagnosis," Leukemia, vol. 4, pp. 3136-3142, 2017.

[60] J. Rawat, A. Singh, and H. S. Bhadauria, "An approach for leukocytes nuclei segmentation based on image fusion," in Proceedings of the 2014 IEEE International Symposium on
Signal Processing and Information Technology (ISSPIT), Noida, India, 2014.

[61] S. Mishra, B. Majhi, P. K. Sa, and L. Sharma, "Gray level cooccurrence matrix and random forest based acute lymphoblastic leukemia detection," Biomedical Signal Processing and Control, vol. 33, pp. 272-280, 2017.

[62] S. Khobragade, D. D. Mor, and C. Y. Patil, "Detection of leukemia in microscopic white blood cell images," in Proceedings of the 2015 International Conference on Information Processing (ICIP), Pune, India, 2015.

[63] G. Singh, G. Bathla, and S. Kaur, "Design of new architecture to detect leukemia cancer from medical images," International Journal of Applied Engineering Research, vol. 11, pp. 70877094, 2016.

[64] V. Singhal and P. Singh, "Texture features for the detection of acute lymphoblastic leukemia," in Proceedings of the 2016 International Conference on ICT for Sustainable Development, Ahmedabad, India, 2016.

[65] L. Zhang, Q. G. Wang, and J. P. Qi, "Processing technology in microscopic images of cancer cells in pleural fluid based on fuzzy edge detection method," Journal of Physics: Conference Series, vol. 48, p. 61, 2006.

[66] S. C. Neoh, W. Srisukkham, L. Zhang et al., “An intelligent decision support system for leukaemia diagnosis using microscopic blood images," Scientific Reports, vol. 5, Article ID 14938, 2015.

[67] M. M. Amin, S. Kermani, A. Talebi, and M. G. Oghli, "Recognition of acute lymphoblastic leukemia cells in microscopic images using k-means clustering and support vector machine classifier," Journal of Medical Signals and Sensors, vol. 5, pp. 49-58, 2015.

[68] K. ElDahshan, M. Youssef, E. Masameer, and M. A. Mustafa, "An efficient implementation of acute lymphoblastic leukemia images segmentation on the FPGA," Advances in Image and Video Processing, vol. 3, p. 8, 2015.

[69] J. Rawat, A. Singh, H. S.. Bhadauria, and J. Virmani, "Computer aided diagnostic system for detection of leukemia using microscopic images," Procedia Computer Science, vol. 70, pp. 748-756, 2015.

[70] S. Mohapatra, D. Patra, and S. Satpathy, "An ensemble classifier system for early diagnosis of acute lymphoblastic leukemia in blood microscopic images," Neural Computing and Applications, vol. 24, no. 7-8, pp. 1887-1904, 2014.

[71] A. A. Nasir, M. Y. Mashor, and R. Hassan, "Classification of acute leukaemia cells using multilayer perceptron and simplified fuzzy ARTMAP neural networks," International Arab Journal of Information Technology, vol. 10, pp. 1-9, 2013.

[72] S. Mohapatra, D. Patra, S. Kumar, and S. Satpathy, "Lymphocyte image segmentation using functional link neural architecture for acute leukemia detection," Biomedical Engineering Letters, vol. 2, no. 2, pp. 100-110, 2012.

[73] H. T. Madhloom, S. A. Kareem, and H. Ariffin, "A robust feature extraction and selection method for the recognition of lymphocytes versus acute lymphoblastic leukemia," in Proceedings of the 2012 International Conference on Advanced Computer Science Applications and Technologies (ACSAT), Kuala Lumpur, Malaysia, 2012.

[74] C. E. Pedreira, L. Macrini, M. G. Land, and E. S. Costa, "New decision support tool for treatment intensity choice in childhood acute lymphoblastic leukemia," IEEE Transactions on Information Technology in Biomedicine, vol. 13, pp. 284290, 2008.

[75] I. Q. Khilji, K. Saha, J. A. Shonon, and M. I. Hossain, “Application of homomorphic encryption on neural network in 
prediction of acute lymphoid leukemia," International Journal of Advanced Computer Science and Applications (IJACSA), vol. 11, no. 6, 2020.

[76] M. Sharif, J. Amin, A. Siddiqa et al., "Recognition of different types of leukocytes using YOLOv2 and optimized bag-offeatures," Emerging Deep Learning Theories and Methods for Biomedical Engineering, vol. 8, pp. 167448-167459, 2020.

[77] H. Fan, F. Zhang, L. Xi, Z. Li, and G. Liu, "LeukocyteMask: an automated localization and segmentation method for leukocyte in blood smear images using deep neural networks," Journal of Biophotonics, vol. 12, no. 7, 2019.

[78] S. Praveena and S. P. Singh, "Sparse-FCM and deep convolutional neural network for the segmentation and classification of acute lymphoblastic leukaemia," Biomedical Engineering/Biomedizinische Technik, vol. 65, no. 6, pp. 759773,2020

[79] S. Shafique and S. Tehsin, "Acute lymphoblastic leukemia detection and classification of its subtypes using pretrained deep convolutional neural networks," Technology in Cancer Research \& Treatment, vol. 17, 2018.

[80] H. C. W. De Vet, J. Koudstaal, W.-S. Kwee, D. Willebrand, and J. W. Arends, "Efforts to improve interobserver agreement in histopathological grading," Journal of Clinical Epidemiology, vol. 48, no. 7, pp. 869-873, 1995.

[81] G. Díaz and A. Manzanera, "Automatic analysis of microscopic images in hematological cytology applications," Clinical Technologies: Concepts, Methodologies, Tools and Applications, pp. 325-52, IGI Global, Philadelphia, PA, USA, 2011.

[82] S. Wan and M.-W. Mak, Machine Learning for Protein Subcellular Localization Prediction, Walter de Gruyter, Berlin, Germany, 2015. 